\title{
Helium Ion Microscopy of Plant Tissues and Mammalian Cells
}

\author{
Andrew F. Loftus ${ }^{1}$, Matthew S. Joens ${ }^{1}$, Sarah E. Dunn ${ }^{1}$, Michael W. Adams ${ }^{1}$, Chuong Huynh ${ }^{2}$, \\ Bernhard Goetze $^{2}$ and James A.J. Fitzpatrick ${ }^{1}$ \\ ${ }^{1}$ Waitt Advanced Biophotonics Center, Salk Institute for Biological Studies, 10010 N. Torrey Pines \\ Road, La Jolla, CA 92037 \\ ${ }^{2}$ Ion Microscopy Innovation Center, Carl Zeiss Microscopy, 1 Corporation Way, Peabody, MA 01960
}

From plants to animals, the helium ion microscope (HIM) facilitates ultrastructural investigations of biological systems at both high contrast and resolution without the need for heavy metal coatings [1]. In this study, we describe not only the advantages of HIM over the low-voltage scanning electron microscopy (LV-SEM) of plant tissues, but will also demonstrate advances in preserving sub-nanometer membrane structures of in vitro cell cultures.

In principle, the operation of a HIM is similar to SEM with the exception that the ion beam has no crossovers. Briefly, a metal tip, forming a three-atom apex is held at a bias in the presence of helium gas, creating a localized ion field. Positively charged ions are subsequently extracted, accelerated, shaped and scanned in the form of a beam across the sample with the resultant secondary electrons collected. The higher mass helium ions give rise to a shorter de Broglie wavelength when compared to the SEM, effectively resulting in an order of magnitude smaller probe size. This higher mass also yields deeper beam penetration prior to scattering which causes less beam-induced damage and enhancement of SE1 secondary electron signal. The HIM has an added benefit in that charging is negated using an electron flood gun interlaced with the scanning ion beam. Compared to SEM, HIM provides the greatest benefit in charge compensation for uncoated samples. Traditionally SEM samples are imaged with a heavy metal coating for conduction and increasing ionization yield, or imaged at low accelerating voltage to reduce charging artifacts. The imaging of uncoated biological samples can be limited in LVSEM by charging as exhibited in the side-by-side evaluation of Arabidopsis thaliana with the HIM (Figure 1). While decreasing the field of view (FOV) in the HIM does not result in any evident loss of resolution, the LV-SEM is plagued by image distortions resulting from charge accumulation. However, both metal coating and low-voltage imaging result in the obstruction of surface features, or a decrease in material contrast and topography at higher resolutions.

With the clear advantages of HIM imaging demonstrated in plant tissues, the surface ultrastructure of in vitro HeLa cell cultures were investigated due to their ubiquity in biological studies. Previous efforts resulted in a wide range of cell membrane irregularities resulting in the loss topological information [2]. Subsequent efforts have centered on attempting to overcome the issue of membrane degradation utilizing a dual approach involving fixation and labeling methods. Briefly, HeLa cells were cultured on coverglass slips, fixed using standard aldehyde treatments, and critical point dried (CPD) as described previously [2]. Some samples were supplemented with 1\% Tannic Acid (TA) and/or osmium fixed prior to the CPD step to enhance membrane integrity. In tandem, additional HeLa cells were incubated with cholera toxin subunit $\mathrm{B}(\mathrm{CTxB})$ to investigate whether $\mathrm{CTxB}$ binding to the saturated lipids present in raft structures might act as a stabilization agent. Briefly, CTxB samples were incubated with CTxBFITC, and were either aldehyde fixed and osmium treated prior to CPD or were immunolabeled with a rabbit anti-cholera primary antibody, followed by a AlexaFluor-594 goat anti-rabbit secondary antibody, aldehyde fixed, osmium treated, and CPD. From the high magnification HIM images (Figure 2), there are clear variations in the effectiveness of each fixation approach to preserve the cellular membrane. 
Osmium fixation prior to CPD allows for a more stable membrane even in the absence of other fixation agents. The use of tannic acid, which is believed to stabilize the unsaturated lipids during fixation with gultaraldehyde [3], resulted in no improvement in the stability of the membranes (Figure 2C). The CTxB-primary-secondary antibody (Figure 2E) samples showed improvement, particularly in the retention of saturated lipids found in caveolar raft structures (Figure 2E) as compared to CTxB with no cross-linking antibody present (Figure 2D).

This work showcases the application of HIM as compared to LV-SEM in its capability to visualize the sub-nanometer ultrastructure of biological systems with both high-resolution and reduced charging artifacts. These results clearly show the enhancement of lipid membrane stability via the use of enhanced fixation and labeling approaches. In addition, the approach of using CTxB protein, known to bind to ganglioside lipids found primarily in cellular rafts along with crosslinking antibodies seems to allow for the retention of those raft components on the cell surface (Figure 2F). We propose a hypothesis of a CTxB protein / antibody network that serves to stabilize the cellular membrane in saturated lipid raft regions where the proteins have associated.

\section{References:}

[1] Bell, D. C. Microscopy and Microanalysis, (2009), 15(2), p.147-153.

[2] MS Joens et al. Scientific Reports, (2013), 3, p. 3514.

[3] Hayat, M.A. "Stains and cytochemical methods", Plenum Press (New York) p.336-339.

[4] The authors acknowledge financial support from the Waitt Foundation, W.M. Keck Foundation, NCI (CA014195) and NINDS (NS072031).

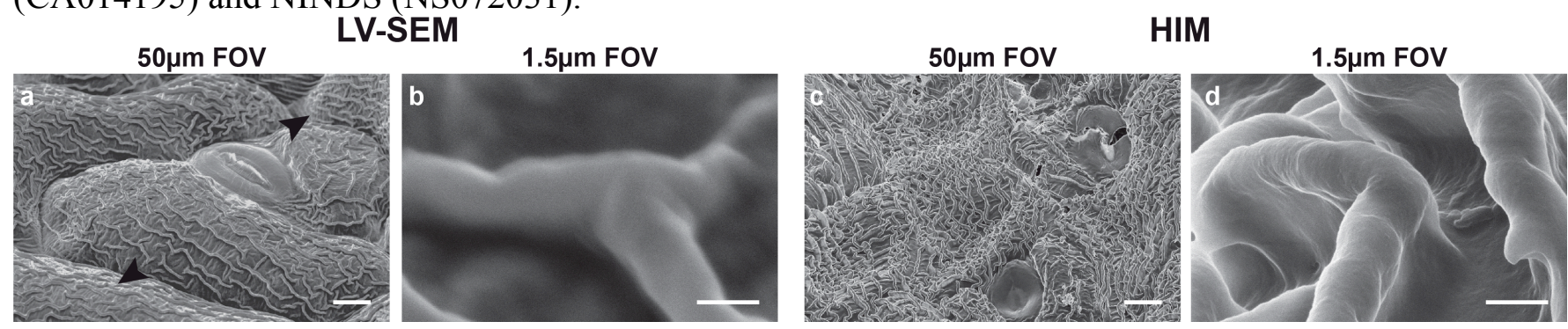

Figure 1. Comparison of SEM and HIM in Arabidopsis thaliana. Increasing magnification series of uncoated and dried $A$. thaliana sepal cuticle in the SEM at $3 \mathrm{KeV}$ (a, b) and HIM (c, d). The difference in resolution is apparent in panels (b) and (d) and the difference in depth of field is apparent at low SEM magnifications as indicated by the black arrowheads in (a). Scale bars: (a,c) $5 \mu \mathrm{m}(\mathrm{b}, \mathrm{d}) 250 \mathrm{~nm}$.
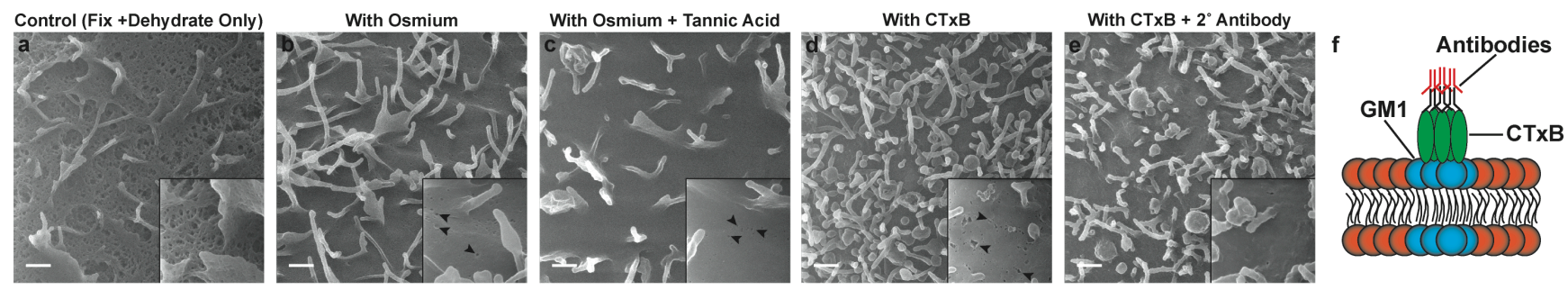

Figure 2. HIM images of HeLa cells with various fixation conditions and schematic of CTxB binding. (A) Aldehyde fixed with CPD. (B) Aldehyde fixed, osmium fixed, with CPD. (C) Fixed as in (B) with $1 \%$ TA supplemented. (D) Incubated with $\mathrm{CTxB}$ and fixed similar to (B). (E) Incubated with $\mathrm{CTxB}$, primary antibody and secondary antibody and fixed similar to (B). (F) Schematic of the binding of CTxB to lipid rafts and antibodies to the CTxB. Black arrows in (B-D) indicate membrane vacancies considered to be caveolar raft structures eliminated of saturated lipids. Scale Bar $=500 \mathrm{~nm}$. 